\title{
Evaluation of the Cytotoxic Effects of the Novel Antineoplastic Agent 1,4,5-Oxathiazinane-4,4- dioxide on Triple Negative Breast Cancer Cells
}

\author{
MARCEL JINIH ${ }^{1}$, JIANG HUAI WANG ${ }^{1}$, ROLF W. PFIRRMANN ${ }^{2}$, \\ D. PETER O'LEARY ${ }^{1}$, MARK A. CORRIGAN ${ }^{1}$ and H. PAUL REDMOND ${ }^{1}$ \\ ${ }^{1}$ Department of Academic Surgery, University College Cork, Cork University Hospital, Cork, Ireland; \\ ${ }^{2}$ Weggis, Lucerne, Switzerland
}

\begin{abstract}
Background/Aim: Adjuvant therapeutic options are limited for triple negative breast cancer (TNBC). Thus, we evaluated the cytotoxic effects of the newly synthesized antineoplastic agent 1,4,5-Oxathiazinane-4,4-dioxide (OTD) on TNBC cells as a potential cancer therapeutic strategy. Materials and Methods: TNBC primary BT-20 and metastatic MDA-MB-231 cell lines were treated with increasing concentrations of OTD for various time periods to assess cell viability. Cell necrosis, apoptosis, necroptosis, autophagy, and ROS generation were evaluated using assay kits or specific inhibitors. Results: Treatment with OTD resulted in a dose-and time-dependent cell death of TNBC BT-20 and MDA-MB-231 cells. OTD also dose-dependently arrested TNBC cell proliferation. Notably, treatment with OTD induced both necrosis and apoptosis of TNBC cells, while the pan-caspase inhibitor Z-VAD-FMK partially attenuated OTD-induced cell death. Importantly, abrogated OTD-induced cell death was observed in the presence of the ROS scavenger $N$-acetylcysteine (NAC), whereas enhanced OTD-induced cell death was observed after the addition of the glutathione synthesis inhibitor BSO, indicating OTDinduced killing of TNBC cells via a reactive oxygen speciesdependent mechanism. Conclusion: OTD is strongly cytotoxic to both primary and metastatic TNBC cells, possibly by inducing multiple cell death pathways.
\end{abstract}

This article is freely accessible online.

Correspondence to: Jiang Huai Wang, MD, Ph.D., Department of Academic Surgery, University College Cork, Cork University Hospital, Cork, Ireland. Tel: +353 214901275, e-mail: jh.wang@ucc.ie; H. Paul Redmond, MCh, FRCSI, Department of Academic Surgery, University College Cork, Cork University Hospital, Cork, Ireland. E-mail: henry.redmond@ hse.ie

Key Words: OTD, triple-negative breast cancer cells, cytotoxicity, cell death, reactive oxygen species.
Breast cancer is the commonest form of cancer in women accounting for approximately $29 \%$ of all new cancer cases and associated with $15 \%$ of cancer-related deaths (1). Of these, triple negative breast cancer (TNBC), characterised by lack of estrogen receptor (ER), progesterone receptor (PR), and human epidermal growth factor receptor type 2 (HER2), is particularly aggressive and carries the worst prognosis. This is largely due to the lack of targeted molecular therapies for TNBC, which is in sharp contrast to ER-positive/PR-positive and HER2-positive subtypes. TNBC also has a poorer outcome after chemotherapy compared to other breast cancer subtypes, reflecting an intrinsically adverse prognosis and aggressive behaviour (2).

Although anti-vascular endothelial growth factor (VEGF)/VEGF receptor (VEGFR) monoclonal antibodies (e.g. bevacizumab), anti-VEGFR tyrosine kinase inhibitors (e.g. sunitinib and sorafenib), anti-endothelial growth factor receptor (EGFR) agents (e.g. cetuximab), poly (ADP ribose) polymerase (PARP) inhibitors (e.g. olaparib and veliparib), mammalian target of rapamycin (mTOR) inhibitors (e.g. everolimus) and Src tyrosine kinase inhibitor (e.g. dasatinib) have been studied in clinical trials, the results are variable (3). The most commonly used treatment regimens for TNBC in the clinical setting in many countries are a combination of anthracyclines, cyclophosphamide, and taxanes, while in some developing countries, the combination of cyclophosphamide-methrotrexate$5 F U(C M F)$ is widely used for economic reasons $(4,5)$. In many of these trials, drug toxicity and severe side effects were a major source of concern, making the search for novel therapeutic agent(s) with reduced systemic toxicity very crucial.

1,4,5-oxathiazinane-4,4-dioxide (OTD) is a newly synthesized compound that has a ring structure similar to taurultam (Figure 1). Taurultam exists in equilibrium with taurolidine in aqueous solution, which is thought to play a crucial role in its mechanisms of action. Previous studies with taurolidine have shown that it exerts anti-inflammatory properties through inhibition of tumour necrosis factor- $\alpha$ (TNF- $\alpha$ ), interleukin (IL)-1, IL-6, and IL-8, and is clinically 


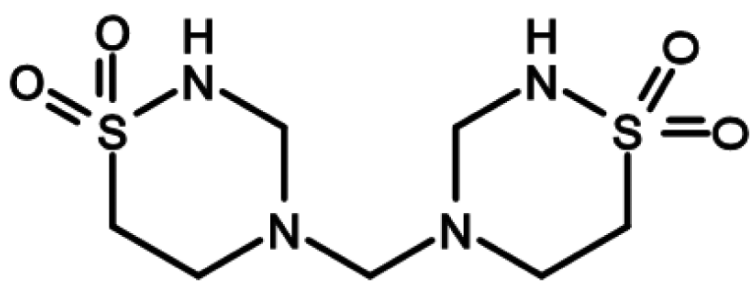

Taurolidine

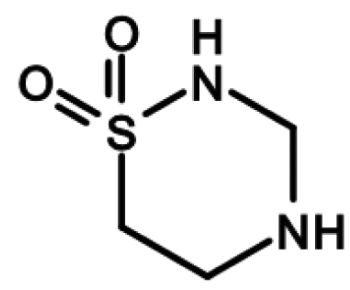

Taurultam

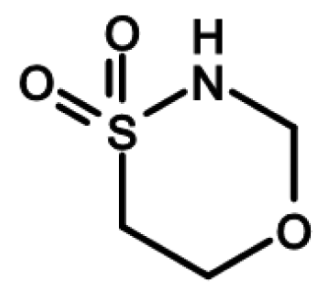

OTD

Figure 1. Molecular structures of taurolidine, taurultam, and 1,4,5-Oxathiazine-4,4-dioxide (OTD).

beneficial in conditions such as abdominal peritonitis and pancreatitis (6-8). From an oncology perspective, taurolidine has been found to exhibit powerful anti-neoplastic properties in colorectal carcinoma (9-11). melanoma (12), glioblastoma (13-16), pancreatic cancer $(17,18)$, and prostate cancer (19), among others. In addition, taurolidine has been shown to inhibit angiogenesis by blocking tumour-derived VEGF (13, 20). Notably, Buchholz et al. have reported that the newly synthesized compound OTD exhibits anti-neoplastic properties in pancreatic cancer cells both in vitro and in vivo, in a dose- and time-dependent manner similar to those of taurolidine/taurultam (21). However, the mechanism by which OTD works is still largely unexplored, and available data are scarce, although preliminary results suggest that OTD may work in a fashion similar to that of taurolidine. A number of studies have demonstrated that taurolidine works by inducing apoptosis, as evidenced by increased DNA fragments in the sub G0/G1 phase of the cell cycle, the release of apoptosis-inducing factor (AIF), and the downregulation of the anti-apoptotic gene $B c l 2(6,11-13)$. It appears that taurolidine induces apoptosis in tumour cells via both intrinsic and extrinsic apoptotic pathways and operates through caspase- and non-caspase-dependent mechanisms as well as by generation of reactive oxygen species (ROS) (13, $16,19,22)$. Interestingly, current data has also shown that OTD not only induces apoptosis and necrosis, but can also initiate the production of ROS in cancer cells (21).

In the clinical setting, taurolidine can be administered to patients through numerous routes including intraperitoneally, intravenously, and topically, and has been shown to exhibit an excellent safety profile $(6,7,14,23,24)$. The only known side effect of taurolidine is peripheral vein irritation when given at high doses intravenously. Allergic reactions have not been observed and are unlikely to happen, because in aqueous solution, taurolidine exists in equilibrium with methylol-taurultam and taurultam, both of which are rapidly metabolised to the physiological substances taurine and carbon dioxide. In comparison, OTD, in addition to intravenous, intraperitoneal, and topical administrations, can be given orally. OTD has a significantly longer half-life of approximately $24 \mathrm{~h}$ compared to the $30 \mathrm{~min}$ of taurolidine. Additionally, OTD also causes less burning pain in open wounds compared with taurolidine (24). The aim of the present study was to evaluate the cytotoxic effects of the novel compound OTD in TNBC cells.

\section{Materials and Methods}

Reagents, cell lines and cultures. The OTD ultra-pure powder, kindly provided by Geistlich Pharma AG (Wolhusen, Switzerland), was dissolved in phosphate-buffered saline (PBS) solution ( $\mathrm{pH} 7.4$ ), filtered through $0.2 \mu \mathrm{m}$ syringe filters (Pall Corporation, Ann Arbor, MI, USA), and prepared to the desired concentrations. Nacetylcysteine (NAC) and DL-buthionine-sulfoximine (BSO) were purchased from Sigma-Aldrich (St. Louis, MO, USA). Z-VADFMK, Necrostatin-1 (Nec-1), and 3-Methyladenine (3-MA) were obtained from InvivoGen (San Diego, CA, USA) and Abcam (Cambridge, MA, USA), respectively. Two types of human TNBC cell lines, BT-20 and MDA-MB-231, were used in the present study. The primary TNBC cell line BT-20 was purchased from American Type Culture Collection (ATCC, Manassas, VA, USA) and cultured in Eagle's minimum essential medium (EMEM) supplemented with $10 \%$ fetal bovine serum (FBS) and $1 \%$ penicillin-streptomycin. The metastatic TNBC cell line MDA-MB-231, isolated from a pleural effusion of a patient with invasive ductal carcinoma, was purchased from ATCC and cultured in DMEM with 10\% FBS and 1\% penicillin-streptomycin. All cells were cultured at $37^{\circ} \mathrm{C}$ in a humidified atmosphere with $5 \% \mathrm{CO}_{2}$ until reaching a sub-confluent monolayer. Sub-confluent cultures of BT-20 and MDA-MB-231 cells were harvested by trypsinization, resuspended in the respective culture medium, and adjusted to the desired cell concentrations for in vitro experiments. All culture media and reagents for cell cultures were purchased from Invitrogen Life Technologies (Paisley, Scotland, UK). All other chemicals, unless indicated, were obtained from Sigma-Aldrich.

Assessment of cell viability and OTD-induced cytotoxicity. Subconfluent cultures of BT-20 and MDA-MB-231 were harvested and re-suspended in respective medium at a density of $1.0 \times 10^{5}$ cells $/ \mathrm{ml}$. A $100 \mu \mathrm{l}$ aliquot of this cell suspension was seeded in 96-well cell culture plates (Sarstedt, Numbrecht, Germany) at a final density of $1.0 \times 10^{4}$ cells/well. Cells were incubated for $12 \mathrm{~h}$ to allow adherence and further incubated with fresh culture medium as the control or treated with at increasing concentrations $(100,200,500,1,000$, and 
$2,000 \mu \mathrm{M})$ of OTD for various time periods. Cell viability and cytotoxicity of OTD were assessed by using the 3-(4,5dimethylthiazol-2-yl)-2,5-diphenyltertazolium bromide (MTT) assay based on the yellow-coloured MTT converted to purple-coloured formazan crystals by viable cells. Briefly, at each time point, $10 \mu \mathrm{l}$ of a $5 \mathrm{mg} / \mathrm{ml}$ MTT solution was added into each well and incubation was protracted for another $2 \mathrm{~h}$ at $37^{\circ} \mathrm{C}$. The supernatant was then removed and $100 \mu \mathrm{l}$ of DMSO was added to each well after washing with PBS. The absorbance of each well was measured using a Micro-titre Plate Reader (Dynex Technologies Inc. Chantilly, VA, USA) at $570 \mathrm{~nm}$ wavelength and the optical density (OD) for each well was recorded. Cells without OTD treatment and culture medium containing no cells were used as the positive and negative controls, respectively. An absorbance two times that of the negative control was considered positive for the presence of viable cells (19). Cell viability was expressed as the percentage (\%) of the control.

Measurement of cell proliferation. The harvested BT-20 and MDAMB-231 cells were seeded in 96-well cell culture plates (Sarstedt) at a density of $1.0 \times 10^{4}$ cells/well and incubated for $12 \mathrm{~h}$ to allow the cells to adhere. Cells were further incubated with culture medium as the control or treated with $100,200,500,1,000$, or $2,000 \mu \mathrm{M}$ OTD for $12 \mathrm{~h}$. BT-20 and MDA-MB-231 cell proliferation was assessed by using the commercially available 5-bromo-2-deoxyuridine (BrdU) labelling and detection kit (Exalpha Biologicals Inc, Shirley, MA, USA) based on the incorporation of the thymidine analogue, BrdU to newly replicated DNA during the synthesis (S) phase of the cell cycle. Cell proliferation was expressed as the percentage (\%) of the control.

FACScan analysis for cell apoptosis and necrosis. The harvested BT-20 and MDA-MB-231 cells were seeded in 24-well cell culture plates (Sarstedt) at a density of $0.5 \times 10^{5}$ cells/well for $12 \mathrm{~h}$ and further incubated with culture medium as the control or treated with different concentrations of OTD for $18 \mathrm{~h}$. Cell apoptosis and necrosis were assessed by using the annexin V/FITC and propidium iodide (PI) assay kit (BD Biosciences, San Jose, CA, USA). Cell apoptosis and necrosis were detected by FACScan analysis using CellQuest software (BD Biosciences) based on that cell apoptosis was quantified through the binding of annexin $\mathrm{V}$ to externalised phosphatidylserine, whereas cell necrosis was simultaneously measured through the binding of PI to fragmented DNA (25). Annexin $\mathrm{V}$ positive cells were considered apoptotic, Annexin $\mathrm{V}$ and PI positive cells were considered late apoptotic and/or necrotic, PI positive cells were identified as necrotic, and Annexin V and PI negative cells were considered viable cells. Cell apoptosis and necrosis were expressed as percentages (\%).

FACScan analysis for autophagy induction. The harvested BT-20 and MDA-MB-231 cells were plated and cultured in 12-well cell culture plates (Sarstedt) at $1.0 \times 10^{5}$ cells/well for $12 \mathrm{~h}$, and further incubated with culture medium as the control or treated with 100 , $200,500,1,000$, or $2,000 \mu \mathrm{M}$ OTD for $18 \mathrm{~h}$. Cells were also treated with $0.5 \mu \mathrm{M}$ rapamycin and $10 \mu \mathrm{M}$ chloroquine for $18 \mathrm{~h}$ as a positive control for autophagy induction. Induction of autophagy was assessed using the Cyto-ID autophagy detection kit (Enzo Life Sciences, Farmingdale, NY, USA) based on that a $488 \mathrm{~nm}$-excitable green fluorescent detection probe which specifically fluoresces in autophagic vesicles and an increase in green signal indicates the accumulation of the probe within the cells arising from enhanced autophagic vesicles. Autophagy induction in BT-20 and MDA-MB231 tumour cells after treatment with OTD was quantified by FACScan analysis using CellQuest software (BD Biosciences) and expressed as mean fluorescence intensity (MFI).

Assessment of OTD-induced cell death after inhibition of ROS generation, cell apoptosis, necroptosis, and autophagy. NAC is a radical scavenger agent that increases the amount of the reducing agent glutathione, whereas BSO is an irreversible inhibitor of the $\gamma$-glutamylcysteine synthase, an enzyme required in the biosynthetic step of glutathione synthesis (26). To evaluate whether ROS contributes to OTD-induced killing of TNBC cells, we treated BT20 and MDA-MB-231 cells with OTD in combination with either NAC or BSO. Briefly, BT-20 and MDA-MB-231 cells were plated and cultured in 96-well cell culture plates (Sarstedt) at a density of $1.0 \times 104$ cells/well for $12 \mathrm{~h}$. Cells were then pre-incubated with either $5.0 \mathrm{mM} \mathrm{NAC}$ or $1.0 \mathrm{mM}$ BSO for $30 \mathrm{~min}$, and further incubated with culture medium as the control or treated with different concentrations of OTD for $24 \mathrm{~h}$. Cell viability was assessed using the MTT assay.

To further examine whether cell apoptosis, necroptosis, and autophagy contribute to OTD-induced cell death in TNBC cells, we treated BT-20 and MDA-MB-231 cells with OTD in combination with the pan-caspase inhibitor Z-VAD-FMK, the potent necroptosis inhibitor Nec-1, or the autophagosome formation inhibitor 3-MA. Briefly, BT-20 and MDA-MB-231 cells were plated and cultured in 96-well cell culture plates (Sarstedt) at a density of $1.0 \times 10^{4}$ cells/well for $12 \mathrm{~h}$. Cells were then pre-incubated with either 5.0 $\mu \mathrm{M}$ Z-VAD-FMK, $1.0 \mu \mathrm{M} \mathrm{Nec-1,} \mathrm{or} 5 \mathrm{mM} 3$-MA for $30 \mathrm{~min}$, and further incubated with culture medium as the control or treated with different concentrations of OTD for $24 \mathrm{~h}$. Cell viability was assessed using the MTT assay.

Statistical analyses. All data are expressed as the mean \pm SD. Statistical analysis was performed using one-way ANOVA or Tukey tests between experimental groups (single agent treatment with different doses and single agent versus combined treatment at various time points) with GraphPad software version 6 (Prism, San Diego, CA, USA). Differences were considered statistically significant when the $p$-value was less than 0.05 .

\section{Results}

OTD induces cell death in TNBC cells in a dose- and timedependent manner. We first treated TNBC cells with different concentrations of OTD to assess OTD-induced cell death using the MTT assay. In the primary TNBC cell line BT-20, treatment with OTD for $24 \mathrm{~h}$ resulted in a dosedependent cell death, as OTD at 200, 500, 1,000, and 2,000 $\mu \mathrm{M}$ induced $25.1 \%, 52.7 \%, 65.6 \%$, and $79.3 \%$ of cell death, respectively, when compared to culture medium-treated BT20 cells $(p<0.05, p<0.01)$ (Figure $2 \mathrm{~A})$. Treatment of the metastatic TNBC cell line MDA-MB-231 with OTD for 24 $\mathrm{h}$ also led to a dose-dependent cell death, as OTD at 100, $200,500,1,000$, and $2,000 \mu \mathrm{M}$ caused $20.9 \%, 50.6 \%$, $55.9 \%, 66.5 \%$, and $79.1 \%$ of cell death, respectively, when compared to culture medium-treated MDA-MB-231 cells $(p<0.05, p<0.01)$ (Figure 2B). 

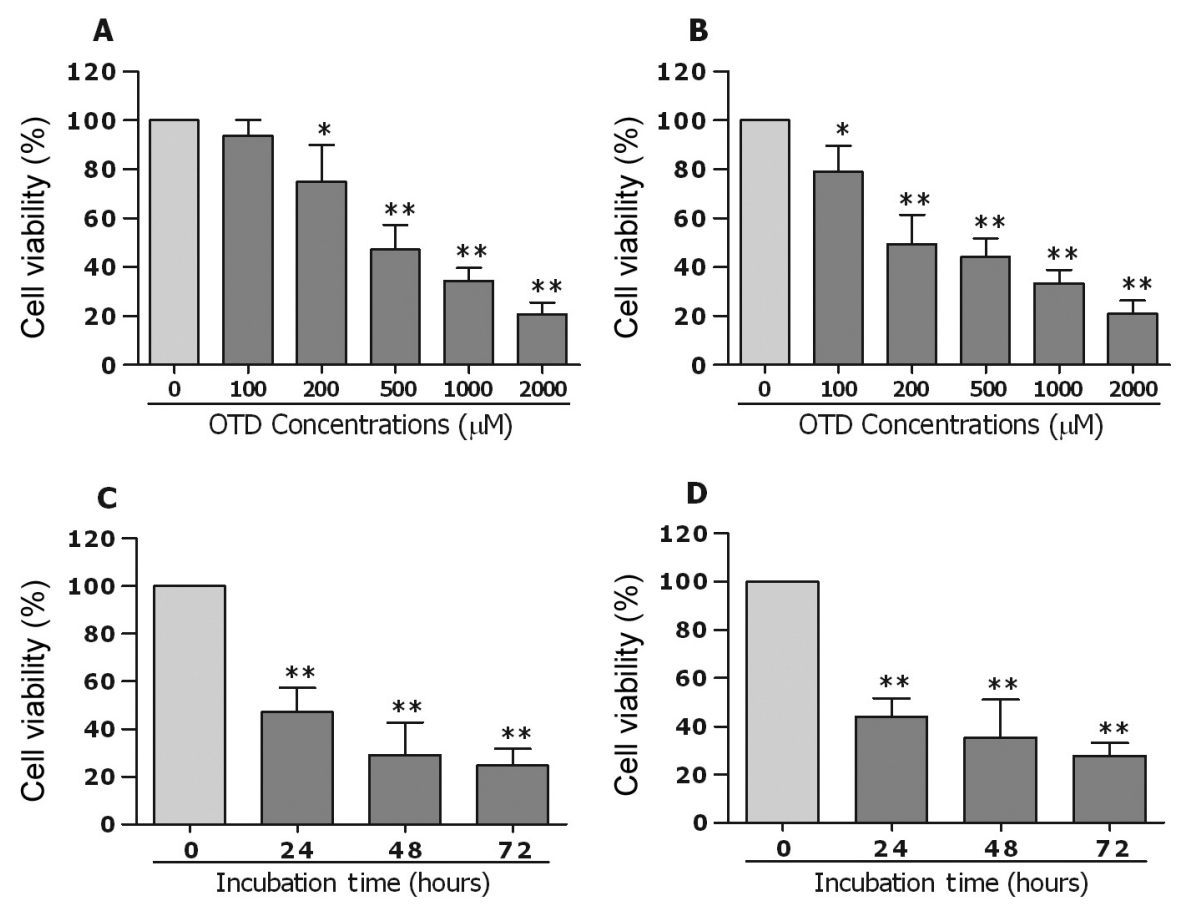

Figure 2. Treatment of triple negative breast cancer (TNBC) cells with OTD induces cell death in a dose-and time-dependent manner. Primary BT20 (A) and metastatic MDA-MB-231 (B) cells were incubated with culture medium as the control or treated with different doses of 1,4,5-Oxathiazine4,4-dioxide (OTD) for 24 h. BT-20 (C) and MDA-MB-231 (D) cells were also treated with $500 \mu M$ OTD for various time periods. Cell viability was assessed using the MTT assay and expressed as the percentage (\%) of the control. Data are expressed as the mean $\pm S D$ from at least five independent experiments in triplicate. ${ }^{*} p<0.05,{ }^{*} p<0.001$ versus $B T-20$ or MDA-MB-231 cells treated with culture medium $(0 \mu M$ OTD).

We next treated TNBC cells with OTD for various time periods. Treatment of primary BT-20 cells with OTD at $500 \mu \mathrm{M}$ for 24 h caused $52.7 \%$ of cell death $(p<0.01$ versus culture medium-treated cells) and a time-dependent increase in cell death was observed in BT-20 cells treated with $500 \mu \mathrm{M}$ OTD for 48 and $72 \mathrm{~h}$ ( $p<0.01$ versus culture medium-treated cells) (Figure 2C). Similar results were also observed in OTD-treated metastatic MDA-MB-231 cells where treatment with $500 \mu \mathrm{M}$ OTD for 24,48 , and $72 \mathrm{~h}$ induced $55.9 \%, 64.7 \%$, and $72.1 \%$ of cell death, respectively ( $p<0.01$ versus culture mediumtreated cells) (Figure 2D).

OTD dose-dependently arrests TNBC cell proliferation. We treated TNBC cells with different doses of OTD for $12 \mathrm{~h}$ to examine the effect of OTD on TNBC cell proliferation using the BrdU cell proliferation assay kit. As shown in Figure 3, incubation of TNBC cells with increasing doses of OTD from $100 \mu \mathrm{M}$ to $2,000 \mu \mathrm{M}$ for $12 \mathrm{~h}$ resulted in a dosedependent reduction in proliferating cells. In BT-20 cells, OTD at the lowest dose of $100 \mu \mathrm{M}$ induced $20.9 \%$ reduction in cell proliferation $(p<0.05$ versus culture medium-treated cells), whereas OTD at $200 \mu \mathrm{M}$ arrested proliferation of $80.3 \%$ cells $(p<0.01$ versus culture medium-treated cells)
(Figure 3A). Of note, at a dose of $500 \mu \mathrm{M}$ and above, OTD almost completely abrogated proliferation of BT-20 cells $(p<0.01$ versus culture medium-treated cells) (Figure 3A). Similarly, in MDA-MB-231 cells, 100 and $200 \mu \mathrm{M}$ OTD caused $16.1 \%$ and $80.6 \%$ reduction in cell proliferation, respectively, compared to culture medium-treated cells $(p<0.05, p<0.01)$ (Figure 3B). At $500 \mu \mathrm{M}$ and above, treatment with OTD for $12 \mathrm{~h}$ arrested proliferation of more than 95\% MDA-MB-231 cells $(p<0.01$ versus culture medium-treated cells) (Figure 3B).

OTD induces both necrosis and apoptosis in TNBC cells. Next, we examined whether treatment of TNBC cells with OTD is capable of inducing cell necrosis and/or apoptosis. TNBC cells were treated with various concentrations of OTD for $18 \mathrm{~h}$, and cell necrosis and apoptosis were assessed using the Annexin V/FITC and PI assay kit. As shown in Figure 4, incubation of the two TNBC cell types with increasing doses of OTD resulted in a substantial reduction in the viable cell proportion, and this reduction in cell viability was paralleled by gradual increases in both necrotic and apoptotic cell proportions as the concentrations of OTD were enhanced. In BT-20 cells, treatment with $100 \mu \mathrm{M}$ OTD for $18 \mathrm{~h}$ induced 

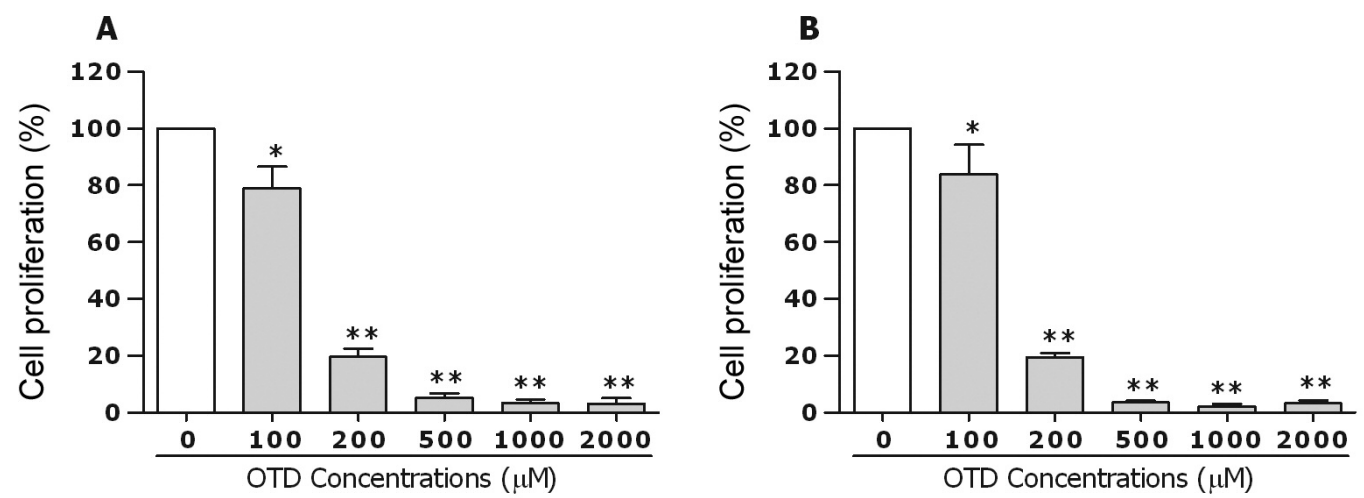

Figure 3. Treatment of triple negative breast cancer (TNBC) cells with 1,4,5-Oxathiazine-4,4-dioxide (OTD) inhibits cell proliferation. Primary BT20 (A) and metastatic MDA-MB-231 (B) cells were incubated with culture medium as the control or treated with different doses of OTD for $12 \mathrm{~h}$. Cell proliferation was analysed using the BrdU labelling and detection kit, and expressed as the percentage (\%) of the control. Data are expressed as the mean $\pm S D$ from four to five independent experiments in triplicate. ${ }^{*} p<0.05$, ${ }^{* *} p<0.001$ versus BT-20 or MDA-MB-231 cells treated with culture medium $(0 \mu M$ OTD $)$.

about $10 \%$ of cell necrosis (Figure 4A). OTD-induced cell necrosis was gradually increased, proportionally to the doses of OTD used and at $1,000 \mu \mathrm{M}$ approximately $34 \%$ of BT-20 cells underwent necrosis (Figure 4A). Treatment with $500 \mu \mathrm{M}$ to $1,000 \mu \mathrm{M}$ OTD induced approximately $9.5 \%$ to $14 \%$ of apoptosis in BT-20 cells after $18 \mathrm{~h}$ incubation (Figure 4A). In MDA-MB-231 cells, a dose-dependent induction of both cell necrosis and apoptosis was also observed after treatment with increasing concentrations of OTD for $18 \mathrm{~h}$ (Figure 4B) where $250 \mu \mathrm{M}$ OTD led to about $11 \%$ of cell necrosis and $4 \%$ of cell apoptosis, while 1,000 $\mu \mathrm{M}$ OTD induced about $29 \%$ of cell necrosis and $12 \%$ of cell apoptosis (Figure 4B).

To further ascertain whether OTD-induced TNBC cell death is partly via induction of cell apoptosis, we used the pan-caspase inhibitor Z-VAD-FMK to block caspasemediated activation of intracellular apoptotic pathways. The addition of $5.0 \mu \mathrm{M}$ Z-VAD-FMK effectively attenuated cell death induced by 500,750 , and $1,000 \mu \mathrm{M}$ OTD for $24 \mathrm{~h}$ in both BT-20 (Figure 4C) and MDA-MB-231 (Figure 4D) cells $(p<0.05, p<0.01$ versus cells treated with OTD alone), suggesting that OTD-induced cell apoptosis is one of the underlying mechanisms responsible for OTD-mediated killing of TNBC cells.

OTD induces cell death in TNBC cells via a ROS-dependent mechanism. NAC is a key component in the biosynthesis of the potent reducing agent glutathione that converts ROS to physiological substances in the body (26). As shown in Figure $5 \mathrm{~A}$ and $\mathrm{B}$, co-treatment of TNBC cells with OTD plus $5.0 \mathrm{mM}$ NAC led to substantial attenuation in OTD-induced cell death. At the concentrations of 500 and $1,000 \mu \mathrm{M}$ of OTD, the addition of $5.0 \mathrm{mM}$ NAC in BT-20 cells reduced OTD-induced cell death by approximately $78 \%$ and $84 \%$, respectively
( $p<0.01$ versus cells treated with OTD alone) (Figure 5A), whereas similar reduction in cell death by NAC was also observed in MDA-MB-231 cells treated with $500 \mu \mathrm{M}$ or 1,000 $\mu \mathrm{M}$ OTD ( $p<0.01$ versus cells treated with OTD alone) (Figure $5 \mathrm{~B})$. On the other hand, co-treatment with OTD and BSO resulted in an increase in OTD-induced cell death in TNBC cells (Figure 5C and D). BSO is an irreversible inhibitor of the $\gamma$-glutamylcysteine synthase, an enzyme required in the biosynthetic step of glutathione synthesis; therefore, BSO administration reduces the amount of available glutathione in the body (26). Of note, the addition of $1.0 \mathrm{mM}$ BSO resulted in significant increases in both $750 \mu \mathrm{M}$ and 1,000 $\mu \mathrm{M}$ OTDinduced death of BT-20 (Figure 5C) and MDA-MB-231 (Figure 5D) cells ( $p<0.05, p<0.01$ versus cells treated with OTD alone). These results indicate that OTD-induced cell death in TNBC cells is predominantly dependent on ROS generation.

OTD fails to induce necroptosis or autophagy in TNBC cells. Nec-1 is a specific inhibitor of the receptor-interacting protein kinase 1 (RIPK3), a primary kinase responsible for cell necroptosis (27); therefore, we used Nec-1 to examine whether the killing of TNBC cells by OTD is associated with OTD-induced cell necroptosis. The addition of $1.0 \mu \mathrm{M} \mathrm{Nec-}$ 1 did not have any significant influence on cell death induced by $100,250,500,750$, and $1,000 \mu \mathrm{M}$ OTD for $24 \mathrm{~h}$ in both BT-20 (Figure 6A) and MDA-MB-231 (Figure 6B) cells, indicating that OTD is unable to initiate necroptosis in these cells.

We further assessed whether OTD induces autophagy in TNBC cells using the Cyto-ID autophagy detection kit. Treatment of BT-20 (Figure 6C) and MDA-MB-231 (Figure 6D) cells with various concentrations of OTD for $18 \mathrm{~h}$ did not cause autophagy formation in these cells. 3-MA is an inhibitor 

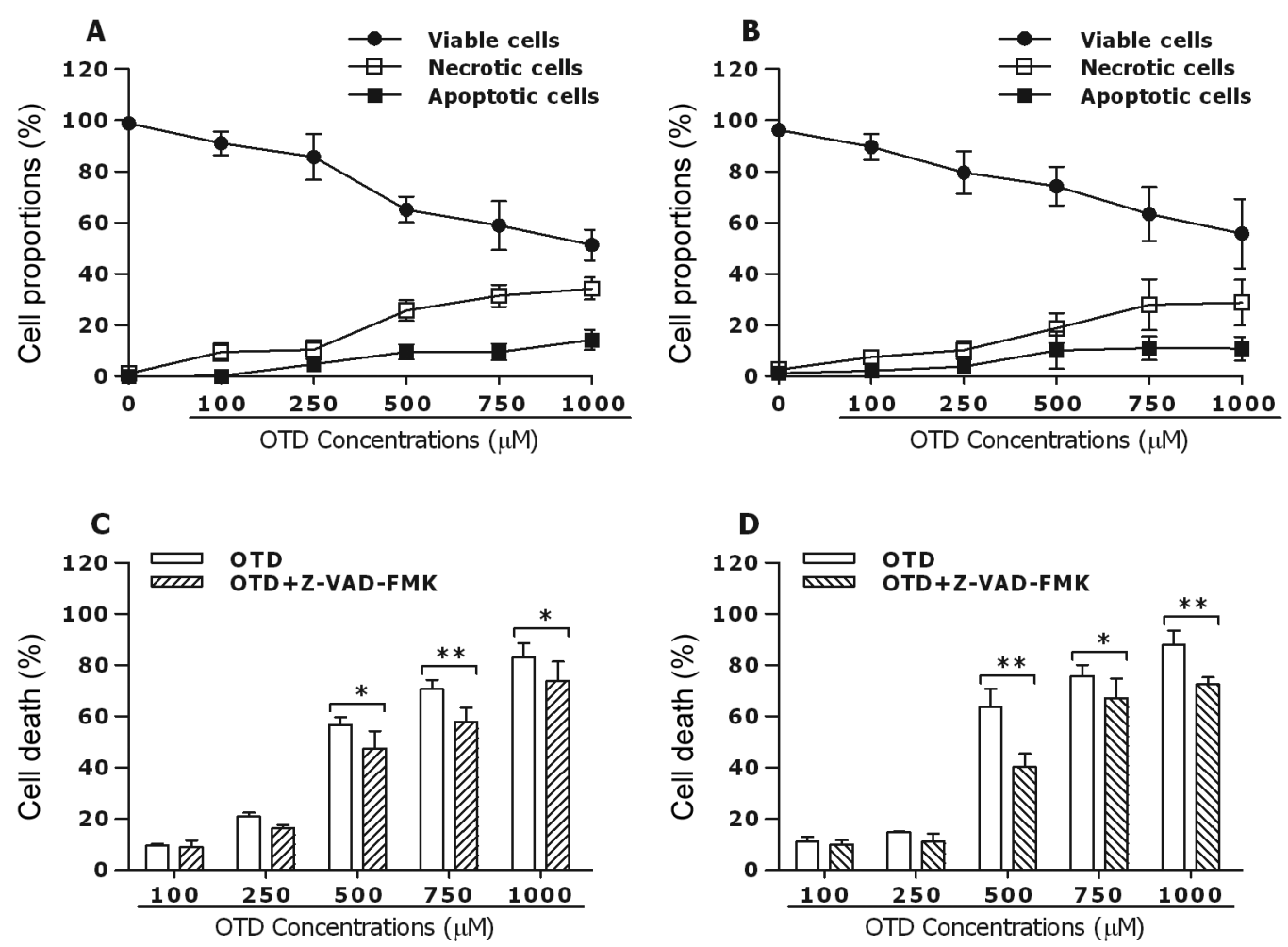

Figure 4. Treatment of triple negative breast cancer (TNBC) cells with 1,4,5-Oxathiazine-4,4-dioxide (OTD) causes both necrosis and apoptosis. Primary BT-20 (A) and metastatic MDA-MB-231 (B) cells were incubated with culture medium as the control or treated with 100, 250, 500, 750, and 1,000 $\mu \mathrm{M}$ OTD for $18 \mathrm{~h}$. The proportion of viable cells, necrotic cells, and apoptotic cells was assessed by FACScan analysis using the annexin V/FITC and PI assay kit, and expressed as percentage (\%). Data are expressed as the mean \pm SD from at least five independent experiments. BT-20 (C) and MDA-MB-231 (D) cells were also pre-incubated with $5.0 \mu M$ Z-VAD-FMK for 30 min before they were treated with different doses of OTD for $24 \mathrm{~h}$. Cell viability was assessed using the MTT assay and expressed as the percentage (\%) of cell death. Data are expressed as the mean $\pm S D$ from three to four independent experiments in triplicate. ${ }^{*} p<0.05$, ${ }^{*} p<0.001$ versus BT-20 or MDA-MB-231 cells treated with OTD alone.

of autophagy that suppresses class III phosphatidylinositol 3kinase (PI3K) activation, thereby preventing the formation of autophagosomes, the characteristic of autophagy (15). The addition of 5.0 mM 3-MA to both BT-20 and MDA-MB-231 cells did not show any significant alterations in OTD-mediated cell killing (data not shown), suggesting that OTD fails to induce autophagy in TNBC cells.

\section{Discussion}

Clinical outcomes of patients with TNBC have not improved significantly over the last decade partly because of the intrinsically aggressive behaviour of this type of tumour and the lack of available targeted therapies $(2,4,5)$. In the present study, we used both TNBC primary BT-20 and metastatic MDA-MB231 cell lines and demonstrated that ex vivo treatment with OTD is effectively cytotoxic in both BT-20 and MDA-MB-231 cells by not only inducing cell death but also arresting cell proliferation. The cytotoxic effect of OTD on TNBC cells as well as the inhibition in cell proliferation as shown in the present study, support the findings by Buchholz et al. who found that OTD exerts strong antineoplastic effects in pancreatic cancer cells (21). In the first part of our study, we assessed cell viability using the MTT assay. This revealed that OTD induced a dose- and time-dependent cell death in TNBC cells. OTD at the low dose of $200 \mu \mathrm{M}$ for example, induced more than $25 \%$ and $50 \%$ of cell death in BT-20 and MDA-MB-231 cells, respectively, after incubation for $24 \mathrm{~h}$. With regards to cell proliferation, incubation of TNBC cells with OTD resulted in significant reduction in proliferating cells in a dose-dependent manner. For instance, incubation with $200 \mu \mathrm{M}$ OTD resulted in cell cycle arrest in approximately $80 \%$ of both BT- 20 and MDA-MB-231 cells. The patterns of OTD-induced cell death and arrest shown in the present study appear to be comparable to taurolidine, based on previous studies (28-30). This may be explained by the metabolism of both OTD and taurolidine, whereby both agents form the common metabolite taurultam, which is an effective antineoplastic agent $(31,32)$. 

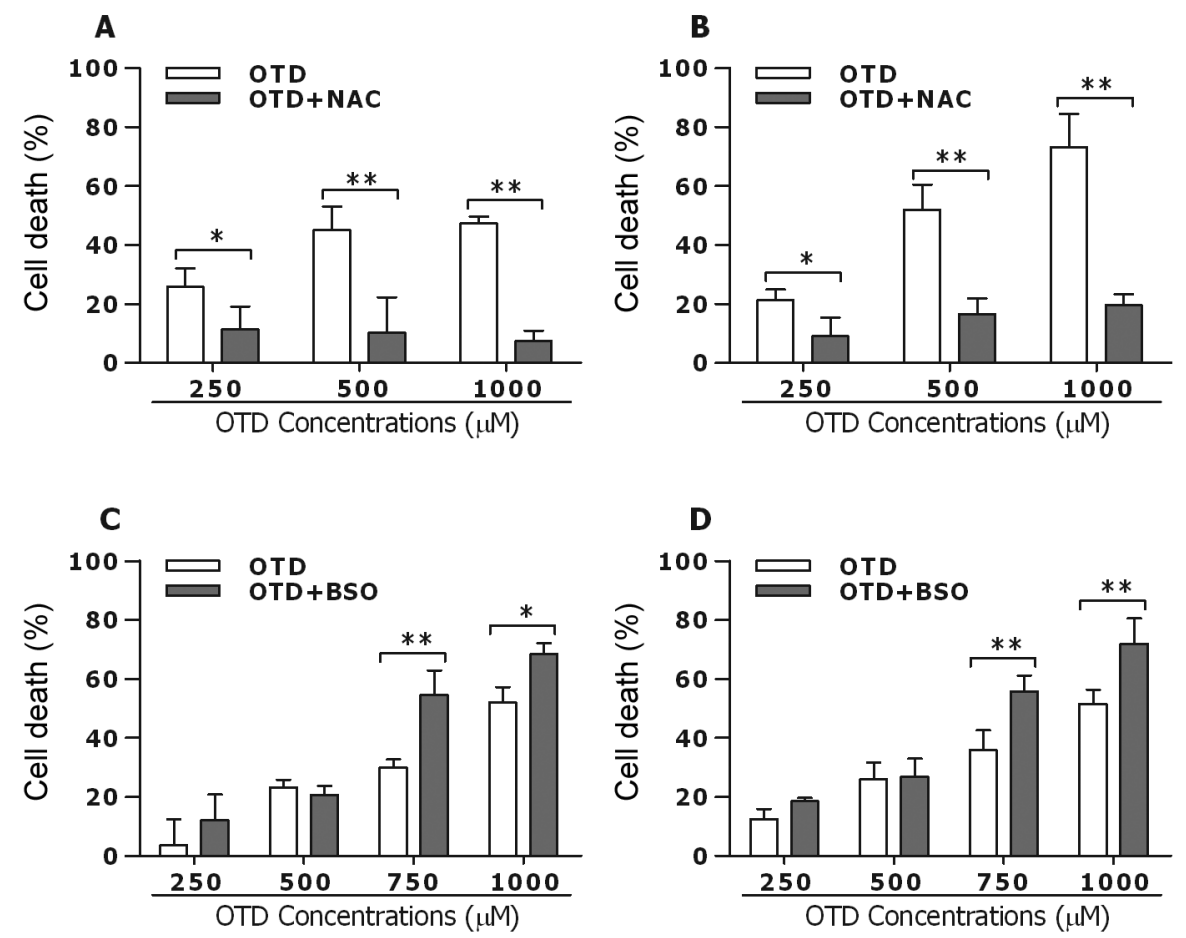

Figure 5. 1,4,5-Oxathiazine-4,4-dioxide (OTD)-induced triple negative breast cancer (TNBC) cell death is dependent on ROS generation. Primary BT-20 (A, C) and metastatic MDA-MB-231 (B, D) cells were pre-incubated with either $5.0 \mathrm{mM} \mathrm{NAC}(A, B)$ or $1.0 \mathrm{mM} \mathrm{BSO}(C, D)$ for $30 \mathrm{~min}$ and further treated with different doses of OTD for $24 \mathrm{~h}$. Cell viability was assessed using the MTT assay and expressed as the percentage (\%) of cell death. Data are expressed as the mean $\pm S D$ from three to five independent experiments in triplicate. ${ }^{*} p<0.05, * * p<0.001$ versus BT-20 or MDA$M B-231$ cells treated with OTD alone.

We further revealed that treatment with OTD led to a dose-dependent increase in the proportions of necrotic and apoptotic BT-20 and MDA-MB-231 cells by FACS analysis using the Annexin V/FITC and PI assay kit. Cell necrosis was observed even following treatment with $100 \mu \mathrm{M}$ for 18 $\mathrm{h}$, which is the lowest dose of OTD and appeared to increase relative to the increasing concentrations of OTD, whereas cell apoptosis was observed only at the higher doses of OTD at 500 and $1,000 \mu \mathrm{M}$, and was absent at low doses. This is somewhat in contrast to the previous studies involving the use of taurolidine, where cell apoptosis was induced by taurolidine even at low doses, between 50 and $100 \mu \mathrm{M}$, depending on cancer cell types $(11,12,33)$.

We next determined the contribution of ROS in OTDmediated killing of TNBC cells. Our results showed that NAC abrogated OTD-induced cell death while BSO, consistent with its induction of ROS, enhanced OTDinduced cell death. In line with this, both primary BT-20 and metastatic MDA-MB-231 cells responded to NAC coincubation with an attenuated cell death; however, the extent of protection offered by NAC was only partial in both TNBC cell lines. Previous studies with taurolidine demonstrated that ROS induced apoptosis via both caspase- dependent and caspase-independent pathways $(7,11,12$, 34). Results from the present study showed an increasing number of late-apoptotic/necrotic cells as revealed by FACScan analysis. The classical apoptosis is strongly related to caspase activation and ROS generation (26). In the present study, although co-treatment with the radical scavenger NAC resulted in an abrogation of OTD-induced killing of TNBC cells, the addition of the pan-caspase inhibitor Z-VAD-FMK only partially attenuated OTDinduced cell killing. These observations are similar to the findings by Stendel et al. on glioblastoma cells (15) and do not fully support the classical apoptosis as the primary mechanism of action for OTD. It is therefore most likely that caspase-independent apoptotic pathways may play a predominant role in the mechanism of action of OTD in TNBC cells. Additionally, Stendel et al. showed that taurolidine induces autophagy and necroptosis in glioblastoma cells, other forms of programmed cell death. However, these two cell death types were not observed in the present study and did not account for the OTD-induced cell death in TNBC cells.

Although the cytotoxic effect of OTD on both BT-20 and MDA-MB-231 cells was well demonstrated in the present 

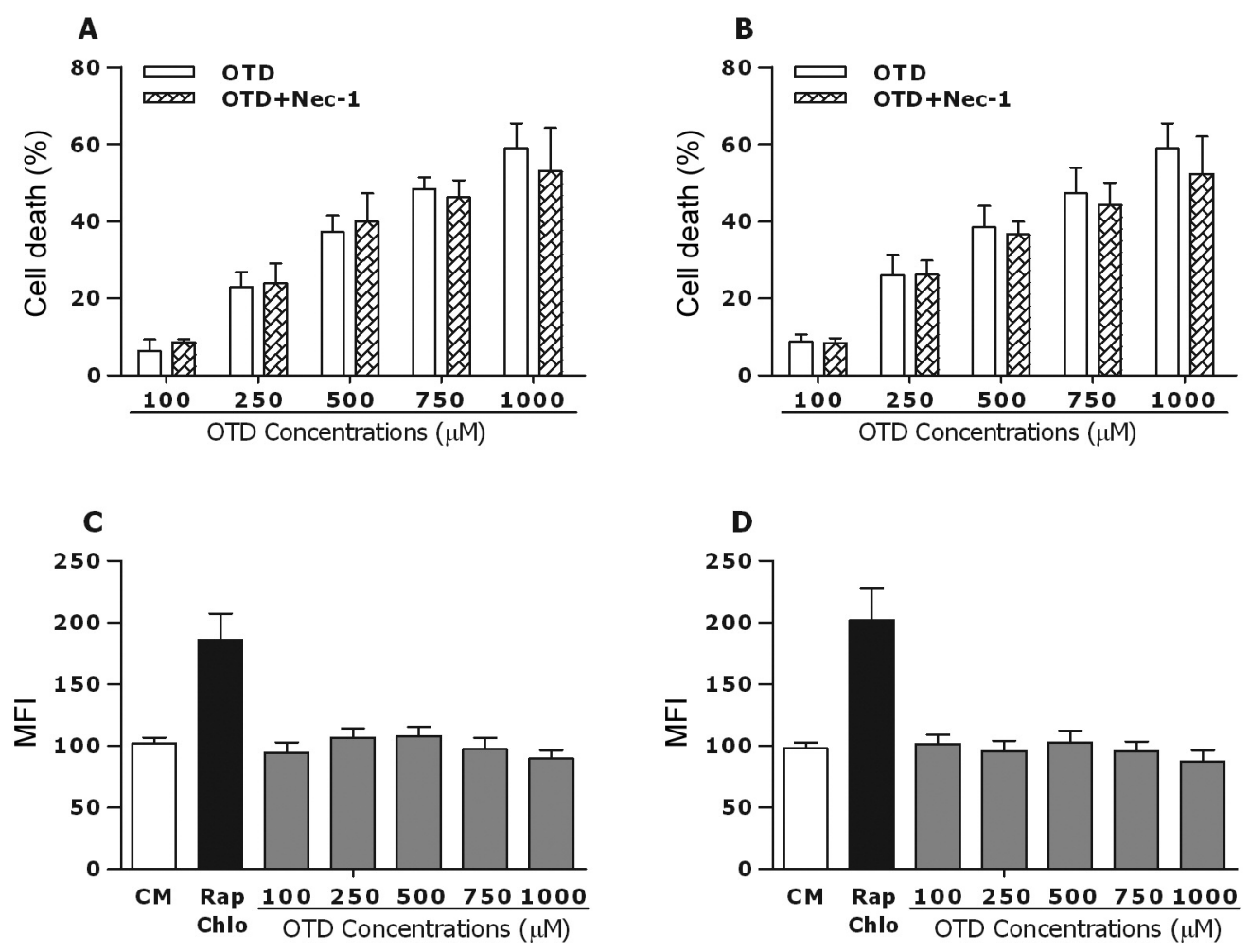

Figure 6. 1,4,5-Oxathiazine-4,4-dioxide (OTD) does not induce necroptosis or autophagy in triple negative breast cancer (TNBC) cells. Primary $B T-20$ (A) and metastatic MDA-MB-231 (B) cells were pre-incubated with $1.0 \mu M$ Nec-1 for 30 min and further treated with different doses of OTD for $24 \mathrm{~h}$. Cell viability was assessed using the MTT assay and expressed as percentage (\%) of cell death. BT-20 cells (C) and MDA-MB-231 (D) cells were incubated with culture medium (CM) as the control or treated with 100, 250, 500, 750, and 1,000 $\mu M$ OTD for 18 h. Induction of autophagy was assessed using the Cyto-ID autophagy detection kit and expressed as mean fluorescence intensity (MFI). Data are mean \pm SD from four to five independent experiments in duplicate or in triplicate.

study, the molecular and genetic pathways involved are still poorly understood. TNBC is associated with several alterations in the molecular pathways that may explain its aggressive behaviour. For instance, TNBC is associated with increased angiogenesis characterised by up-regulated expression of VEGFR $(3,35)$. Previous work demonstrated that taurolidine not only reduces the synthesis of VEGF, but also potently inhibits VEGF-induced angiogenesis from human endothelial cells in vitro $(7,36)$. TNBC is also associated with PI3KCA mutations (35), and inhibitors of the PI3K/Akt/mTOR pathway have been shown to be effective in killing breast cancer cells $(37,38)$. Previous work involving mesothelioma cells showed that taurolidine inactivates Akt and its downstream p70 S6 kinase (p70S6K) effector in a dose- and time-dependent manner (29). These antineoplastic properties of taurolidine, a compound of OTD, may partly explain why OTD is a potent antineoplastic agent in TNBC cells.

Taken together, treatment with OTD in vitro induces significant cell death in both primary and metastatic TNBC cells. Our data demonstrate that OTD works through the induction of multiple cell death pathways, and primarily via inducing necrosis and apoptosis. OTD-associated ROS generation in TNBC cells may play a fundamental role in its mode of action. These encouraging ex vivo results of OTDinduced killing of TNBC cells merit further investigation in vivo and in patients with TNBC in the perioperative neoadjuvant and adjuvant setting.

\section{Conflicts of Interest}

Dr. Rolf W. Pfirrmann is a retired medical director in Geistlich Pharma AG.

\section{Authors' Contributions}

M.J., J.H.W., R.W.P., and H.P.R. designed the study, M.J. and J.H.W. performed experiments, M.J., J.H.W., D.P.O., and M.A.C. analyzed data, M.J., J.H.W., and H.P.R. wrote the manuscript, and all Authors reviewed and approved the manuscript. 


\section{Acknowledgements}

The Authors thank Siobhan Blankson and Qiong Di Wu (Department of Academic Surgery, University College Cork) for their technical assistance. 1,4,5-Oxathizinane-4,4-dioxide (OTD) was provided and granted by Geistlich Pharma AG, Wolhusen, Switzerland.

\section{References}

1 Siegel RL, Miller KD and Jemal A: Cancer statistics, 2015. CA Cancer J Clin 65(1): 5-29, 2015. PMID: 25559415. DOI: $10.3322 /$ caac. 21254

2 Foulkes WD, Smith IE and Reis-Filho JS: Triple-negative breast cancer. N Engl J Med 363(20): 1938-1948, 2010. PMID: 21067385. DOI: 10.1056/NEJMra1001389

3 Crown J, O'Shaughnessy J and Gullo G: Emerging targeted therapies in triple-negative breast cancer. Ann Oncol 23(Suppl 6): vi56-vi65, 2012. PMID: 23012305. DOI: 10.1093/annonc/ mds 196

4 Moreno-Aspitia A and Perez EA: Anthracycline- and/or taxaneresistant breast cancer: Results of a literature review to determine the clinical challenges and current treatment trends. Clin Ther 31(8): 1619-1640, 2009. PMID: 19808124. DOI: 10.1016/j.clinthera.2009.08.005

5 Bhattacharya R, Banerjee K, Mukherjee N, Sen M and Mukhopadhyay A: From molecular insight to therapeutic strategy: The holistic approach for treating triple negative breast cancer. Pathol Res Pract 213(3): 177-182, 2017. PMID: 28215644. DOI: $10.1016 /$ j.prp.2017.01.001

6 Neary PM, Hallihan P, Wang JH, Pfirrmann RW, BouchierHayes DJ and Redmond HP: The evolving role of taurolidine in cancer therapy. Ann Surg Oncol 17(4): 1135-1143, 2010. PMID: 20039217. DOI: 10.1245/s10434-009-0867-9

7 Möhler H, Pfirrmann RW and Frei K: Redox-directed cancer therapeutics: Taurolidine and Piperlongumine as broadly effective antineoplastic agents (review). Int J Oncol 45(4): 13291336, 2014. PMID: 25175943. DOI: 10.3892/ijo.2014.2566

8 Browne MK, Leslie GB and Pfirrmann RW: Taurolin, a new chemotherapeutic agent. J Appl Bacteriol 41(3): 363-368, 1976. PMID: 828157. DOI: 10.1111/j.1365-2672.1976.tb00647.x

9 McCourt M, Wang JH, Sookhai S and Redmond HP: Taurolidine inhibits tumor cell growth in vitro and in vivo. Ann Surg Oncol 7(9): 685-691, 2000. PMID: 11034247. DOI: 10.1007/s10434000-0685-6

10 Nestler G, Schulz HU, Schubert D, Krüger S, Lippert H and Pross M: Impact of taurolidine on the growth of CC531 coloncarcinoma cells in vitro and in a laparoscopic animal model in rats. Surg Endosc 19(2): 280-284, 2005. PMID: 15870965. DOI: $10.1007 / \mathrm{s} 00464-003-9301-8$

11 Chromik AM, Daigeler A, Hilgert C, Bulut D, Geisler A, Liu V, Otte JM, Uhl W and Mittelkötter U: Synergistic effects in apoptosis induction by taurolidine and TRAIL in HCT-15 colon carcinoma cells. J Invest Surg 20(6): 339-348, 2007. PMID: 18097875. DOI: 10.1080/08941930701772157

12 Sun BS, Wang JH, Liu LL, Gong SL and Redmond HP: Taurolidine induces apoptosis of murine melanoma cells in vitro and in vivo by modulation of the Bcl-2 family proteins. J Surg Oncol 96(3): 241248, 2007. PMID: 17469118. DOI: 10.1002/jso.20827
13 Rodak R, Kubota H, Ishihara H, Eugster HP, Könü D, Möhler H, Yonekawa $\mathrm{Y}$ and Frei K: Induction of reactive oxygen intermediatesdependent programmed cell death in human malignant ex vivo glioma cells and inhibition of the vascular endothelial growth factor production by taurolidine. J Neurosurg 102(6): 1055-1068, 2005. PMID: 16028765. DOI: 10.3171/jns.2005.102.6.1055

14 Stendel R, Picht T, Schilling A, Heidenreich J, Loddenkemper C, Jänisch W and Brock M: Treatment of glioblastoma with intravenous taurolidine. First clinical experience. Anticancer Res 24(2C): 1143-1147, 2004. PMID: 15154639.

15 Stendel R, Biefer HR, Dékány GM, Kubota H, Münz C, Wang $\mathrm{S}$, Mohler H, Yonekawa $\mathrm{Y}$ and Frei $\mathrm{K}$ : The antibacterial substance taurolidine exhibits anti-neoplastic action based on a mixed type of programmed cell death. Autophagy 5(2): 194-210, 2009. PMID: 19066471. DOI: 10.4161/auto.5.2.7404

16 Stendel R, Stoltenburg-Didinger G, Al Keikh CL, Wattrodt M and Brock M: The effect of taurolidine on brain tumor cells. Anticancer Res 22(2A): 809-814, 2002. PMID: 12014655.

17 Braumann C, Gutt CN, Scheele J, Menenakos C, Willems W, Mueller JM and Jacobi CA: Taurolidine reduces the tumor stimulating cytokine interleukin-1beta in patients with resectable gastrointestinal cancer: a multicentre prospective randomized trial. World J Surg Oncol 7: 32, 2009. PMID: 19309495. DOI: 10.1186/1477-7819-7-32

18 Chromik AM, Hahn SA, Daigeler A, Flier A, Bulut D, May C, Harati K, Roschinsky J, Sülberg D, Weyhe D, Mittelkötter U and Uhl W: Gene expression analysis of cell death induction by taurolidine in different malignant cell lines. BMC Cancer 10: 595, 2010. PMID: 21034493. DOI: 10.1186/1471-2407-10-595

19 Darnowski JW, Goulette FA, Cousens LP, Chatterjee D and Calabresi P: Mechanistic and antineoplastic evaluation of taurolidine in the DU145 model of human prostate cancer. Cancer Chemother Pharmacol 54(3): 249-258, 2004. PMID: 15173956. DOI: 10.1007/s00280-004-0806-1

20 Braumann C, Tangermann J, Jacobi CA, Müller JM and Dubiel W: Novel anti-angiogenic compounds for application in tumor therapy - COP9 signalosome-associated kinases as possible targets. Mini Rev Med Chem 8(5): 421-428, 2008. PMID: 18473931. DOI: 10.2174/138955708784223549

21 Buchholz M, Majchrzak-Stiller B, Hahn S, Vangala D, Pfirrmann RW, Uhl W, Braumann $\mathrm{C}$ and Chromik AM: Innovative substance 2250 as a highly promising anti-neoplastic agent in malignant pancreatic carcinoma - in vitro and in vivo. BMC Cancer 17(1): 216, 2017. PMID: 28340556. DOI: 10.1186/s12885-017-3204-x

22 Han Z, Ribbizi I, Pantazis P, Wyche J, Darnowski J and Calabresi P: The antibacterial drug taurolidine induces apoptosis by a mitochondrial cytochrome c-dependent mechanism. Anticancer Res 22(4): 1959-1964, 2002. PMID: 12174870.

23 Redmond HP, Neary PM, Jinih M, O'Connell E, Foley N, Pfirrmann RW, Wang JH and O'Leary DP: randomised clinical trial assessing use of an anti-inflammatory agent in attenuating peri-operative inflammation in non-metastatic colon cancer - the S.U.R.G.U.V.A.N.T. trial. BMC Cancer 18(1): 794, 2018. PMID: 30081854. DOI: 10.1186/s12885-018-4641-x

24 Kichko TI, Pfirrmann RW and Reeh PW: Taurolidine and congeners activate hTRPA1 but not hTRPV1 channels and stimulate CGRP release from mouse tracheal sensory nerves. Pharmacol Res Perspect 4(1): e00204, 2016. PMID: 26977296. DOI: $10.1002 /$ prp2.204 
25 Vermes I, Haanen C, Steffens-Nakken H and Reutelingsperger C: A novel assay for apoptosis. Flow cytometric detection of phosphatidylserine expression on early apoptotic cells using fluorescein labelled Annexin V. J Immunol Methods 184(1): 3951, 1995. PMID: 7622868. DOI: 10.1016/0022-1759(95)00072-i

26 Simon HU, Haj-Yehia A and Levi-Schaffer F: Role of reactive oxygen species (ROS) in apoptosis induction. Apoptosis 5(5): 415418, 2000. PMID: 11256882. DOI: 10.1023/a:1009616228304

27 Linkermann A and Green DR: Necroptosis. N Engl J Med 370(5): 455-465, 2014. PMID: 24476434. DOI: 10.1056/ NEJMra1310050

28 Nici L, Monfils B and Calabresi P: The effects of taurolidine, a novel antineoplastic agent, on human malignant mesothelioma. Clin Cancer Res 10(22): 7655-7661, 2004. PMID: 15569998. DOI: 10.1158/1078-0432.CCR-0196-03

29 Aceto N, Bertino P, Barbone D, Tassi G, Manzo L, Porta C, Mutti L and Gaudino G: Taurolidine and oxidative stress: A rationale for local treatment of mesothelioma. Eur Respir J 34(6): 1399-1407, 2009. PMID: 19460788. DOI: 10.1183/090 31936.00102308

30 Imhof L, Goldinger SM, Baumann K, Schad K, French LE, Röthlisberger P and Dummer R: The antibacterial substance, taurolidine in the second/third-line treatment of very advanced stage IV melanoma including brain metastases: Results of a phase 2, open-label study. Melanoma Res 21(1): 80-83, 2011. PMID: 21052029. DOI: 10.1097/CMR.0b013e328341442d

31 Gong L, Greenberg HE, Perhach JL, Waldman SA and Kraft WK: The pharmacokinetics of taurolidine metabolites in healthy volunteers. J Clin Pharmacol 47(6): 697-703, 2007. PMID: 17395893. DOI: $10.1177 / 0091270007299929$

32 Hood HT, Smail GA, Skellern GG, Jindal DP, Browne MK and Pfirrmann RW: Studies of the thiadiazine, taurolidine-I. Identification of the molecular species present in aqueous solutions by (1)H- and (13)C-NMR spectroscopy. Talanta 41(1): 107-113, 1994. PMID: 18965894. DOI: 10.1016/0039-9140(94) $80175-4$
33 Chromik AM, Daigeler A, Hilgert C, Bulut D, Geisler A, Liu V, Otte JM, Uhl W and Mittelkötter U: Synergistic effects in apoptosis induction by taurolidine and TRAIL in HCT-15 colon carcinoma cells. J Invest Surg 20(6): 339-348, 2007. PMID: 18097875. DOI: $10.1080 / 08941930701772157$

34 Walters DK, Muff R, Langsam B, Gruber P, Born W and Fuchs B: Taurolidine: A novel anti-neoplastic agent induces apoptosis of osteosarcoma cell lines. Invest New Drugs 25(4): 305-312, 2007. PMID: 17458504. DOI: 10.1007/s10637-007-9052-9

35 Le Du F, Eckhardt BL, Lim B, Litton JK, Moulder S, MericBernstam F, Gonzalez-Angulo AM and Ueno NT: Is the future of personalized therapy in triple-negative breast cancer based on molecular subtype? Oncotarget 6(15): 12890-12908, 2015. PMID: 25973541. DOI: 10.18632/oncotarget.3849

36 Jacobi CA, Menenakos C and Braumann C: Taurolidine - a new drug with anti-tumor and anti-angiogenic effects. Anticancer Drugs 16(9): 917-921, 2005. PMID: 16162968. DOI: 10.1097/ 01.cad.0000176502.40810.b0

37 Sangai T, Akcakanat A, Chen H, Tarco E, Wu Y, Do KA, Miller TW, Arteaga CL, Mills GB, Gonzalez-Angulo AM and MericBernstam F: Biomarkers of response to Akt inhibitor MK-2206 in breast cancer. Clin Cancer Res 18(20): 5816-5828, 2012. PMID: 22932669. DOI: 10.1158/1078-0432.CCR-12-1141

38 Dey N, De P and Leyland-Jones B: PI3K-AKT-mTOR inhibitors in breast cancers: From tumor cell signaling to clinical trials. Pharmacol Ther 175: 91-106, 2017. PMID: 28216025. DOI: 10.1016/j.pharmthera.2017.02.037
Received March 2, 2021

Revised March 19, 2021

Accepted March 22, 2021 